\title{
Revisión sistemática de los instrumentos y recursos didácticos de creatividad desarrollados en habla hispana
}

\author{
Alba González Moreno*1; María del Mar Molero Jurado*2 \\ ${ }^{1} \mathrm{https} / / /$ orcid.org/0000-0003-2254-7456, Universidad de Almería, Almería, España, ${ }^{2} \mathrm{https://orcid.org/0000-0001-9187-1474,}$ \\ Universidad de Almería, Almería, España
}

Citar como: González Moreno, A., \& Molero Jurado, M. (2021). Revisión sistemática de los instrumentos y recursos didácticos de creatividad desarrollados en habla hispana. Revista Digital de Investigación en Docencia Universitaria, 15(2), e1440. https://doi. org/10.19083/10.19083/ridu.2021.1440

Recibido: 11/02/2021. Revisado: 31/05/2021. Aceptado: 04/06/2021. Publicado: 26/11/2021

\section{Resumen}

Introducción: La creatividad es una capacidad que está presente en todos los seres humanos y se caracteriza por generar soluciones originales ante un determinado conflicto. Objetivo: Esta revisión sistemática tiene como objetivo identificar tanto los instrumentos para medir la creatividad como los distintos recursos que pueden ser utilizados para su mejora en habla hispana. Método: Para ello se han realizado dos búsquedas: una para instrumentos y otra para recursos, en las bases de datos Web of Science, Scopus, Dialnet Plus y Ebsco, mediante unas determinadas fórmulas de búsqueda, de las cuales se han obtenido un total de 34 estudios. Resultados: Los resultados muestran una diversidad de instrumentos y de recursos, técnicas o actividades existentes en habla hispana con los que se puede potenciar la creatividad. Discusión: Este trabajo puede ayudar a otras investigaciones a identificar cuáles son los instrumentos o recursos que mejor se adaptan a sus estudios por realizar. Palabras clave: creatividad; instrumento de medida; recursos educativos abiertos; programas de educación; español.

\section{Systematic review of creativity didactic tools and resources developed in spanish}

\begin{abstract}
Introduction: Creativity is a capacity that is present in all human beings and that is characterized by generating original solutions to a certain conflict. Objective: This systematic review aims to identify both the instruments to measure creativity and the different resources that can be used to improve it in Spanish. Method: For this, two searches have been carried out: one for instruments and another for resources in the Web of Science, Scopus, Dialnet Plus and Ebsco databases using certain search formulas, from which a total of 34 studies have been obtained from among both searches. Results: The results show a diversity of instruments and resources, techniques or activities existing in the Spanish language with which creativity can be enhanced. Discussion: This work can help other researches to identify which instruments or resources are best adapted to their studies to be carried out.
\end{abstract}

Keywords: creativity; measuring instruments; open educational resources; educational programmes; spanish. 


\section{Introducción}

El concepto de creatividad es bastante complejo que resulta difícil de definir, ya que no existe una definición general para dicho término (Al-Ababneh, 2020). Aun así, la creatividad puede ser entendida como la capacidad que mediante la imaginación construye algo nuevo y que tiene transcendencia en el mundo físico, así como todo proceso que lleva consigo una toma de decisiones con el fin de cumplir un objetivo específico (Cuetos et al., 2020).

Algunos investigadores como Castillo-Delgado et al. (2016) y Bonilla (2015) indican que la creatividad está relacionada con la transformación social y con el potencial humano. Esta idea se enlaza con el posicionamiento de Fernández et al. (2019) sobre la creatividad como una capacidad para resolver problemas mediante el lanzamiento de hipótesis y la discusión de resultados.

La creatividad es una característica del ser humano que, desde sus inicios, siempre se ha encontrado presente en mayor o menor medida. En los últimos años, concretamente en la última década, este vocablo ha captado el interés de los investigadores de manera significativa (Fernández et al., 2019; Morales-Valiente, 2017). Algunos de los estudios explican cómo las estrategias de aprendizaje y la creatividad favorecen el procesamiento de la información (Hurtado et al., 2018); cómo la creatividad se muestra en las edades más tempranas y cómo esta puede ser fomentada a través de diversas estrategias educativas (Medina-Sánchez et al., 2017); cómo aquellos alumnos que presentan actitudes creativas tienen mayores calificaciones académicas (la creatividad influye de manera directa con el rendimiento académico) (Chiecher et al., 2018); cómo la creatividad se puede llevar a cabo mediante diferentes herramientas pedagógicas y está se relaciona de manera significativa con el aprendizaje (Bernabeu \& Goldstein, 2016; Rodríguez, 2018); cómo la creatividad es una alternativa didáctica con la que se puede potenciar el talento humano de diferentes disciplinas (Acosta et al., 2016).

Todos estos estudios son solo algunos ejemplos de la gran cantidad de investigaciones que se pueden encontrar hoy en día sobre creativi- dad. Gran parte de estos estudios se desarrollan en contextos educativos; aunque como comenta Elisondo (2015), la creatividad es una capacidad que se encuentra inmersa en todas las personas y por ello puede desenvolverse en cualquier situación y/o contexto.

La evaluación de la creatividad es una tarea difícil debido a la ambigüedad de este término, que implica diferentes rasgos de personalidad y de cognición. Este tema es discutido por distintos autores, ya que algunos indican que la creatividad no es un constructo que pueda ser medido, mientras otros señalan que sí se puede medir y que además se debe hacer con el fin de mejorarla mediante una intervención (Arévalo et al., 2015; Sánchez et al., 2019).

Cabe la necesidad de añadir que la subjetividad y la experiencia juegan un papel relevante en el proceso de la valoración de la capacidad creativa de una persona (González et al., 2020). Un aspecto a tener en cuenta es que los estudios experimentales y/o empíricos llevados a cabo por los investigadores pueden verse afectados o alterados negativamente por distintas variables como el ambiente o la intención del investigador, lo que puede poner en riesgo la validez de los datos obtenidos (Ricci, 2020; Rodríguez \& Luzardo, 2020). Se entiende que la habilidad de ser creativo puede ser modificada según una diversidad de factores (sociales, psicológicos, ambientales o biológicos) (Díaz \& Justel, 2019).

Existen diferentes métodos para medir la creatividad, entre los más comunes se pueden encontrar las pruebas de pensamiento divergente que miden la creatividad como una habilidad para crear nuevas ideas ante un determinado problema (Guilford, 1950; Torrance, 1966).

Las dimensiones para la evaluación de la creatividad varían según el ámbito al que se pretende evaluar o incluso depende del punto de vista de cada investigador. La mayoría de los test sobre creatividad coinciden en que la fluidez, la originalidad y la flexibilidad son las tres dimensiones que se deben tener en cuenta a la hora de medir la creatividad, por lo que cuanto mayor sea la puntuación del sujeto en estas dimensiones, mayor será su capacidad creativa (Soto et al., 2015). Por otro lado, otros autores, concretamente Kim et al. 
(2006) discrepan de esta idea, ya que, para ellos, estas tres dimensiones se relacionan entre sí $\mathrm{y}$, por ello, no es necesario que una persona deba tener puntuaciones en las tres dimensiones para que sea creativa. Martínez (2016) indica que, para saber si una persona es creativa, hay que fijarse en el resultado y/o producto y en la utilidad que éste tiene; y no tanto en el proceso de cómo esa persona ha llegado a dicho resultado. Atendiendo a la gran cantidad de tipos de creatividad que pueden existir según el contexto al que se hace referencia, Ayala-Pérez (2013) muestra que, en diseño gráfico, las dimensiones que se deben tener en cuenta son la novedad, la comunicación y la estética, las cuales forman el constructo de la creatividad en esta área y, por ello, con ellas se puede conocer si una persona es creativa o no.

Ante lo expuesto, el objetivo principal de esta revisión sistemática consiste en identificar los instrumentos o adaptaciones existentes que sirven para medir la creatividad, así como distintos recursos o programas desarrollados para su mejora en habla hispana.

\section{Metodología}

Para el desarrollo de este trabajo se han tenido en cuenta los principios de la declaración PRISMA (Urrútia \& Bonfil, 2010) y las pautas metodológicas ofrecidas para la elaboración de revisiones sistemáticas de calidad (Alexander, 2020).

\section{Bases de datos, descriptores y fórmulas de búsqueda establecidos}

Para la presente revisión sistemática se han consultado las bases de datos Web of Science, Scopus, Dialnet Plus y Ebsco en el buscador llamado Google Académico. Este informe, atendiendo a su objetivo principal, ha sido desarrollado mediante dos búsquedas distintas: una primera búsqueda enfocada a los instrumentos que miden creatividad y una segunda búsqueda independiente con el objetivo de identificar diferentes recursos o programas de intervención existentes sobre creatividad.

En la primera búsqueda (en adelante, búsqueda 1), se han utilizado como descriptores creatividad y español. Estos términos han sido posteriormente traducidos al inglés con el fin de ser utilizados en las fórmulas de búsqueda (creativity AND spanish). Esta traducción se debe a que las bases de datos consultadas tienen un carácter internacional, por lo que una búsqueda en inglés siempre será más efectiva, aunque dicha búsqueda ha sido realizada en inglés y español. Las fórmulas de búsqueda empleadas han contado con la ayuda del operador booleano AND con el fin de enriquecer la búsqueda. Por tanto, se ha llevado a cabo la búsqueda mediante las siguientes formulas: creatividad AND español; creativity AND spanish.

Con la segunda búsqueda (búsqueda 2), que ha estado enfocada a los estudios relacionados con programas o recursos de intervención de creatividad, se ha contado con los descriptores programa, intervención y creatividad. Dichos descriptores han sido más adelante traducidos al inglés con la finalidad de establecer una serie de fórmulas de búsqueda apropiadas. Así pues, las fórmulas de búsqueda empleadas para esta segunda indagación han sido en español: programa AND creatividad; intervención AND creatividad; y en inglés: program AND creativity; intervention AND creativity.

Para ambas búsquedas se ha utilizado una serie de filtros tales como el tipo de documento o el idioma utilizado con el propósito de aumentar la eficiencia de la búsqueda. No se ha establecido un límite temporal.

En la tabla 1 se puede observar el número de documentos encontrados en cada una de las bases de datos empleadas para esta revisión sistemática. Dicha tabla está organizada en dos tipos de búsqueda: instrumentos (búsqueda 1) y programas o recursos de intervención (búsqueda 2); cada una de estas búsquedas ha sido realizada en inglés y en español a través de las distintas fórmulas de búsqueda comentadas anteriormente. Tras llevar a cabo la búsqueda inicial, se han propuesto unos criterios de inclusión y exclusión con el fin de seleccionar aquellos estudios que más se adaptan a los objetivos de la presente revisión. En ambas búsquedas (búsqueda 1 y búsqueda 2) se ha contado con los mismos criterios de selección, pero siempre adaptados a su temática.

Para comenzar, haciendo referencia a los criterios de inclusión propuestos, se ha seleccionado 
Tabla 1

Resumen de los resultados obtenidos en cada base de datos atendiendo a la búsqueda realizada

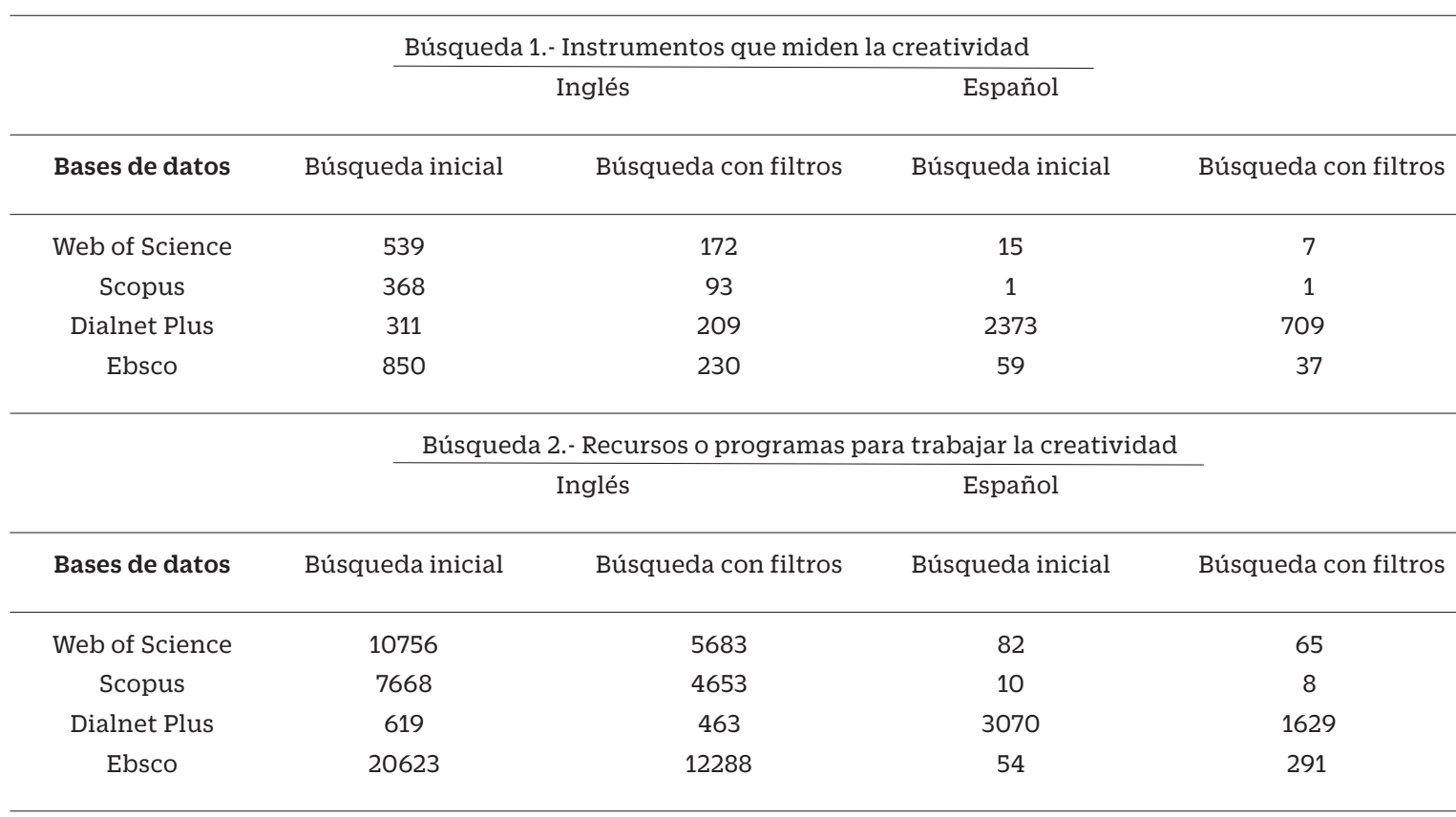

aquellos documentos dotados de los siguientes aspectos: (a) disponer del texto completo; (b) estudios que han sido categorizados como artículos de revista; (c) idioma de publicación español o inglés; (d) estudios de corte empírico; (e) trabajos enfocados a población hispanohablante. Por otro lado, se ha establecido una serie de criterios de exclusión mediante los que se ha decidido si formaran parte de la revisión sistemática o no; dichos criterios han sido: (a) los trabajos catalogados como libro, capítulo de libro, tesis y/o trabajos de fin de estudios; (b) estudios de corte cualitativo o teóricos; (c) investigaciones enfocadas a otra población diferente a hispanohablantes.

\section{Categorización de las publicaciones}

Haciendo referencia a los criterios comentados anteriormente se ha incluido un total de 34 artículos en esta revisión sistemática entre ambas búsquedas.

Por un lado, atendiendo a la búsqueda 1, se encontró inicialmente un total de 4,535 resultados. Estos resultados, tras la aplicación de los criterios de selección, se vieron reducidos a la cantidad de 2,849. Esta cifra disminuyó considerablemente tras la revisión del tema y el contenido de los estudios, dando lugar así a los ocho artículos in- cluidos finalmente en la revisión con respecto a la búsqueda enfocada a los instrumentos de medición de creatividad.

En cuanto a la búsqueda 2, enfocada a recursos que trabajen la creatividad, el número total de resultados encontrados en la búsqueda inicial ha sido 43,376. Una vez aplicados los criterios de exclusión, dicha cifra quedó mermada a 25,080, de los cuales, tras su previa revisión en cuanto a resumen y título se seleccionaron un total de 26 artículos (Figura 1).

Dos investigadoras han sido las que han llevado a cabo el proceso de la búsqueda de documentos, así como, se han encargado de analizar de manera individual tanto el título como el resumen con el fin de seleccionar solo aquellos estudios que atendían el objetivo propuesto. Las discrepancias que han podido surgir han sido resueltas mediante consenso, sin necesidad de recurrir a la participación de terceros.

\section{Resultados}

Atendiendo al propósito de esta revisión sistemática, el apartado de resultados va a verse desglosado en dos. Por un lado, se van a conocer cuáles 
son los instrumentos de habla hispana existentes que miden la creatividad y, por otra parte, cuáles son los programas o recursos didácticos que se han llevado a cabo para la mejora de la misma.

La siguiente tabla (Tabla 2) muestra algunas de las principales características de los estudios seleccionados relacionados con la medición de la creatividad. En dichos estudios se puede destacar como el tipo de medición predominante consiste en una escala tipo Likert con varias opciones de respuesta.

La Escala de Acciones Creativas (Elisondo, 2020) evalúa la cantidad de acciones creativas ejecutadas por los sujetos en siete dominios establecidos (creatividad cotidiana, participación social, música, artes escénicas, ciencia y tecnología, artes plásticas y oficios y, por último, literatura). Dicha escala está destinada a estudiantes, al igual que la elaborada por Soroa et al. (2015) denominada Inventario de Creatividad Emocional breve, que tiene como objetivo evaluar la capacidad de expresar y experimentar emociones mediante tres dimensiones (preparación, novedad y efectividad). Otro de los instrumentos que se puede llevar a cabo con sujetos de estas edades es la Prueba de Creatividad de Villa y Auzmendi (Auzmendi et al. 1996) también conocida como VAT (Villa and Auzmendi Creativity Test). Este instrumento consiste en que, mediante 20 adjetivos, los sujetos deben de autocalificarse mediante una escala Likert de cinco puntos.

Por otro lado, enfocada a edades inferiores, concretamente a alumnos de educación primaria (6-12 años), se encuentra el Test de Creatividad Infantil (TCI) realizado por Romo et al. (2016) con

\section{Figura 1}

Diagrama de flujo del proceso de la selección de la muestra

\section{Búsqueda 1}

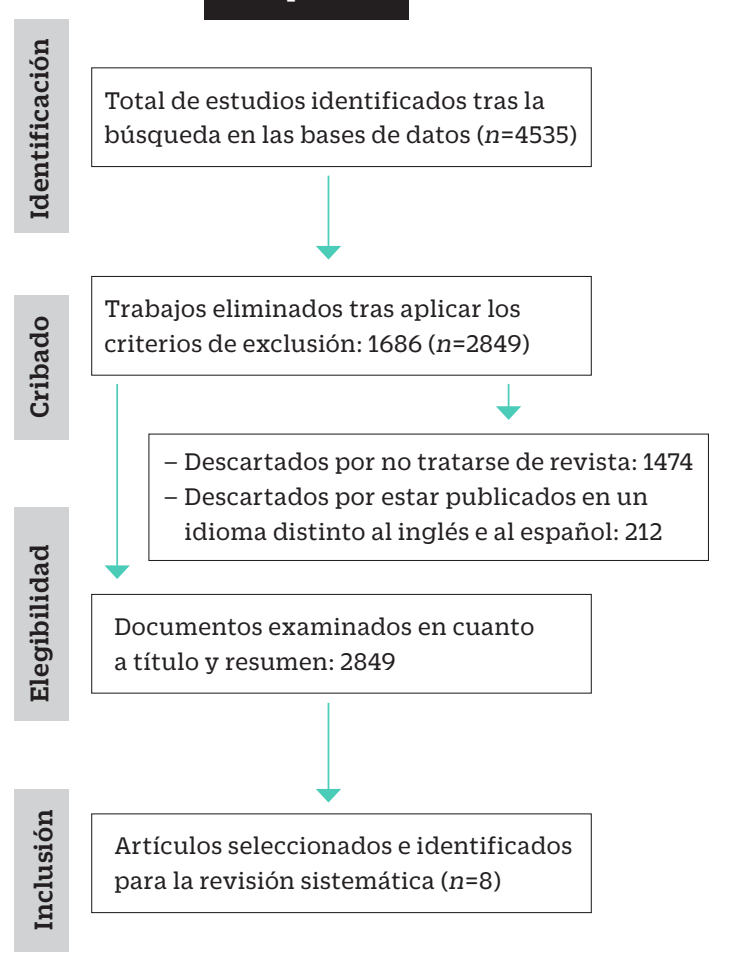

\section{Búsqueda 2}

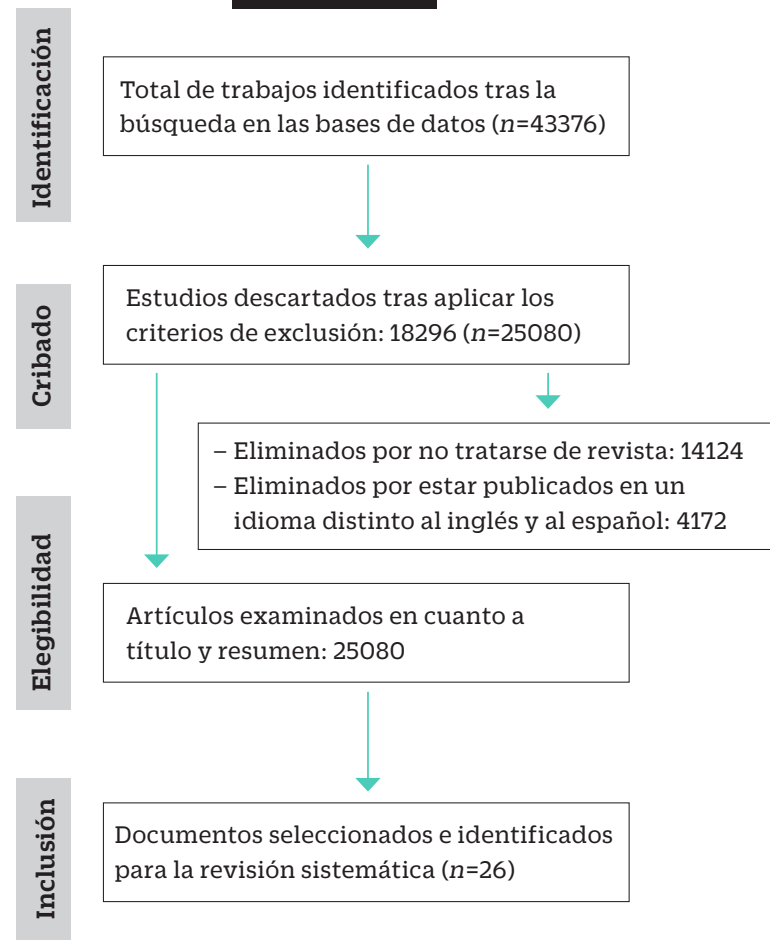

Nota. La figura situada a la izquierda (Búsqueda 1) hace referencia al diagrama de flujo relacionado con la búsqueda de instrumentos, mientras que la figura situada a la derecha (Búsqueda 2) indica la búsqueda relacionada con los recursos educativos. 
el que se evalúa la creatividad a través de dos tareas enfocadas al proceso mediante el cual se obtiene el producto final y no solo en el resultado.

Una de las escalas que ha sido desarrollada para poder evaluar la creatividad en todas las edades posibles es el instrumento CREA (Martínez-Zaragoza \& Corbalán, 2002). Este test está compuesto por un total de tres láminas ( $\mathrm{A}, \mathrm{B}$ y $\mathrm{C}$ ) y sus consiguientes hojas de respuesta. Las láminas A y B están planteadas para ser administradas en sujetos mayores de 16 años, por lo que pueden ser utilizadas de manera conjunta. Por el contrario, la lámina C está diseñada para su administración a niños de edades comprendidas entre 6 y 16 años.

\section{Tabla 2}

Principales características de los estudios sobre instrumentos

\begin{tabular}{|c|c|c|c|c|}
\hline Autor/es y año & $\begin{array}{l}\text { Nombre del } \\
\text { instrumento }\end{array}$ & $\begin{array}{l}\text { Tamaño de la } \\
\text { muestra }\end{array}$ & $\mathrm{N}^{\circ}$ de ítems & Tipo de medición \\
\hline Elisondo (2020) & $\begin{array}{l}\text { Escala de Acciones } \\
\text { Creativas }\end{array}$ & $\begin{array}{l}1604 \text { mujeres y } \\
\text { hombres } \\
(M=37.75)\end{array}$ & $\begin{array}{l}42 \text { ítems con } \\
\text { seis ítems para } \\
\text { cada dominio }\end{array}$ & $\begin{array}{l}\text { Escala Likert con } 5 \\
\text { opciones de respuesta }\end{array}$ \\
\hline Romo et al. (2016) & $\begin{array}{l}\text { Test de Creatividad } \\
\text { Infantil (TCI) }\end{array}$ & $\begin{array}{l}1488 \text { estudiantes de } \\
\text { educación primaria }\end{array}$ & - & Dibujo libre \\
\hline Soroa et al. (2015) & $\begin{array}{l}\text { Inventario de } \\
\text { Creatividad Emocional } \\
\text { - versión abreviada al } \\
\text { español (ECI-S) }\end{array}$ & $\begin{array}{l}1145 \text { estudiantes de } \\
\text { educación superior } \\
(\mathrm{M}=21.70)\end{array}$ & 17 ítems & $\begin{array}{l}\text { Escala Likert de } 6 \\
\text { puntos (1= muy en } \\
\text { desacuerdo - } \\
6=\text { muy de acuerdo) }\end{array}$ \\
\hline $\begin{array}{l}\text { Boada-Grau et al. } \\
\text { (2014) }\end{array}$ & $\begin{array}{l}\text { Escala de Percepción } \\
\text { del Entorno Creativo } \\
\text { (CEP) - Adaptación al } \\
\text { español }\end{array}$ & $\begin{array}{l}975 \text { adultos } \\
\text { empleados } \\
(\mathrm{M}=42.49)\end{array}$ & 9 ítems & $\begin{array}{l}\text { Escala Likert con } 5 \\
\text { opciones de respuesta } \\
(1=\text { completamente } \\
\text { en desacuerdo }-5= \\
\text { completamente de } \\
\text { acuerdo }\end{array}$ \\
\hline $\begin{array}{l}\text { Boada-Grau et al. } \\
\text { (2014) }\end{array}$ & $\begin{array}{l}\text { Escala de Potencial } \\
\text { Creativo y Creatividad } \\
\text { Practicada (CPPC-17) - } \\
\text { Adaptación al español }\end{array}$ & $\begin{array}{l}975 \text { empleados } \\
\text { españoles } \\
(\mathrm{M}=42.49)\end{array}$ & 17 ítems & $\begin{array}{l}\text { Escala Likert con } 5 \\
\text { opciones de respuesta } \\
(1=\text { completamente } \\
\text { en desacuerdo }-5= \\
\text { completamente de } \\
\text { acuerdo }\end{array}$ \\
\hline $\begin{array}{l}\text { Barraca et al. } \\
\text { (2011) }\end{array}$ & $\begin{array}{l}\text { Prueba de Imaginación } \\
\text { Creativa para Adultos } \\
\text { (PIC-A) }\end{array}$ & $\begin{array}{l}488 \text { adultos } \\
(\mathrm{M}=25.7)\end{array}$ & 13 ítems & $\begin{array}{l}4 \text { tareas: } 3 \text { de naturaleza } \\
\text { narrativa y una gráfica }\end{array}$ \\
\hline $\begin{array}{l}\text { Martínez- } \\
\text { Zaragoza y } \\
\text { Corbalán (2002) }\end{array}$ & Instrumento CREA & $\begin{array}{l}2223 \text { sujetos: } 1075 \\
\text { españoles y } 1148 \\
\text { argentinos }\end{array}$ & - & $\begin{array}{l}3 \text { láminas (A, B y C) } \\
\text { según la edad de los } \\
\text { sujetos }\end{array}$ \\
\hline $\begin{array}{l}\text { Auzmendi et al. } \\
\text { (1996) }\end{array}$ & $\begin{array}{l}\text { Prueba de Creatividad } \\
\text { Villa y Auzmendi }\end{array}$ & $\begin{array}{l}2270 \text { estudiantes }(\mathrm{M}= \\
14.2)\end{array}$ & 20 ítems & $\begin{array}{l}\text { Escala Likert con } 5 \\
\text { opciones de respuesta } \\
(1=\text { nada }-5=\text { mucho })\end{array}$ \\
\hline
\end{tabular}


Para adultos se puede encontrar la Prueba de Imaginación Creativa para Adultos (PIC-A) (Barraca et al., 2011), la cual tiene como objetivo identificar la creatividad narrativa y la creatividad gráfica de los sujetos. Para ello, dicho instrumento consta de cuatro tareas: tres de naturaleza narrativa y una gráfica. En la primera de estas tareas los sujetos deben apuntar las ideas que les surjan sobre lo que pueda estar sucediendo en un dibujo que se les presenta; en la tarea dos se tienen que comentar todos los usos que se le puede dar a un tubo de goma; la tarea tres consiste en inventar todas las respuestas posibles ante una situación inconcebible; para finalizar, la tarea cuatro consiste en completar unos dibujos que se presentan incompletos e idear un posible título.

Por último, orientado también a edades adultas, se encuentran dos instrumentos que han sido adaptados al español. En primer lugar, la Escala de Percepción del Entorno Creativo (CEP) (Boada-Grau et al., 2014b) se basa en conocer cuáles son las percepciones que tienen los empleados en cuanto a la creatividad en el trabajo mediante tres escalas: soporte a la creatividad, características del trabajo y bloqueos a la creatividad. Por otro lado, se encuentra la Escala de Potencial Creativo y Creatividad Practicada (CPPC-17) (Boada-Grau et al., 2014ª), la cual se encuentra constituida por tres factores: potencial creativo, creatividad practicada y percepción de apoyo organizacional.

Una vez comentados los artículos provenientes de instrumentos para medir la creatividad se va a dar paso a tratar aquellos trabajos relacionados con programas o recursos elaborados para trabajar la creatividad.

Mediante la observación de la siguiente tabla (Tabla 3) se muestran los resultados encontrados en esta revisión sistemática sobre aquellos recursos, materiales y/o programas que pueden llevarse a cabo con la finalidad de trabajar la creatividad dentro del contexto educativo o de cualquier otro ámbito.

Un aspecto a destacar es cómo la mayoría de estos estudios están enfocados a trabajar la creatividad mediante elementos relacionados con la inteligencia y los procesos cognitivos y, por ello, se llevan a cabo tareas o actividades que impliquen la proposición de nuevas ideas, la resolución de conflictos (Alfonso-Benlliure et al., 2013; Allueva, 2002; López \& Navarro-Lozano, 2010; Prieto et al., 2002; Larraz \& Allueva, 2012) o la utilización de recursos educativos orientados a la construcción de productos como es el Tangram (Piraquive-Peña et al., 2015). A partir de dichas tareas se pretende no solo fomentar la creatividad como tal sino desarrollar en los sujetos unas habilidades creativas adecuadas tales como fluidez, originalidad y flexibilidad.

Otro de los recursos que sobre todo con sujetos de corta edad se suele utilizar es el juego. Mediante el juego o actividades lúdicas basadas en la experimentación los niños no solo obtienen un aprendizaje significativo, sino que a su vez se potencian ciertas habilidades que conllevan una mejora de la creatividad gráfica, narrativa y motriz (Aranda, 2012; Castillo et al., 2018; Chávez et al. 2009; Garaigordobil, 2006; Garaigordobil \& Berrueco, 2011; Justo \& Franco, 2008; Ocampo et al., 2019). Por ello, ciertos estudios se enfocan en trabajar la creatividad mediante sesiones de educación física o ciertas actividades relacionadas que fomenten el movimiento como pueden ser los distintos tipos de desplazamientos (Martínez \& Díaz, 2006; Salinas-López et al., 2015).

Por otro lado, otras de las técnicas de gran utilidad para el desarrollo de la creatividad son aquellas que están relacionadas con las artes plásticas como puede ser pintar sobre un lienzo o construir diferentes estructuras mediante materiales como la plastilina y la arcilla (Garaigordobil \& Pérez, 2002; Jenaro-Río et al., 2019). A partir de dichas técnicas de carácter técnico y visual, los sujetos trabajan ciertas variables como la percepción o la imaginación, las cuales ayudan a estimular la creatividad. Estas últimas variables también pueden verse representadas en aquellos recursos que implican actividades relacionadas con la lectura o escritura de cuentos (Barrena \& Molina, 2010; Franco, 2004; Segundo et al., 2020), el visionado de cortometrajes o series infantiles (Franco \& Justo, 2010; Núñez-Gómez et al., 2020) o aquellos ejercicios orientados a la relajación creativa (Arazola-Ruano, 2017; Franco et al., 2009; Franco \& Justo, 2009), ya que, como se ha comentado anteriormente, mediante dichas variables se puede potenciar la creatividad de manera positiva. 
Tabla 3

Estudios enfocados a programas o recursos para trabajar la creatividad

\begin{tabular}{|c|c|c|c|}
\hline Autor/es y año & Variables a trabajar & Recursos utilizados & $\begin{array}{l}\text { Etapa } \\
\text { educativa }\end{array}$ \\
\hline $\begin{array}{l}\text { Núñez-Gómez et al. } \\
\text { (2020) }\end{array}$ & Creatividad & Cortometrajes infantiles & Primaria \\
\hline Segundo et al. (2020) & $\begin{array}{l}\text { Pensamiento creativo y } \\
\text { rendimiento académico }\end{array}$ & Actividades de lectura y escritura & Primaria \\
\hline Ocampo et al. (2019) & Habilidades creativas & $\begin{array}{l}\text { Ocho juegos infantiles modificados } \\
\text { para promover las habilidades } \\
\text { creativas }\end{array}$ & Primaria \\
\hline Jenaro-Río et al. (2019) & Creatividad gráfica y narrativa & Taller de artes plásticas & Primaria \\
\hline Castillo et al. (2018) & Creatividad & $\begin{array}{l}\text { Programa: ¿Te atreves a crear? - } \\
\text { Actividades lúdicas y colectivas }\end{array}$ & Infantil \\
\hline Arazola-Ruano (2017) & Creatividad motriz & $\begin{array}{l}\text { Actividades basadas en relajación } \\
\text { creativa }\end{array}$ & Infantil \\
\hline $\begin{array}{l}\text { Salinas-López et al. } \\
\text { (2015) }\end{array}$ & Creatividad motriz & $\begin{array}{l}\text { Unidad didáctica enfocada al área de } \\
\text { educación física }\end{array}$ & Primaria \\
\hline $\begin{array}{l}\text { Piraquive-Peña et al. } \\
\text { (2015) }\end{array}$ & $\begin{array}{l}\text { Creatividad, inteligencias } \\
\text { múltiples y motivación }\end{array}$ & Tangram & Secundaria \\
\hline Olivos et al. (2013) & Creatividad gráfica y narrativa & Programa "Yo aprendo y emprendo" & Secundaria \\
\hline $\begin{array}{l}\text { Alfonso-Benlliure et al. } \\
\text { (2013) }\end{array}$ & Creatividad & $\begin{array}{l}\text { Actividades enfocadas al fomento de } \\
\text { procesos cognitivos }\end{array}$ & Infantil \\
\hline Aranda (2012) & Creatividad & $\begin{array}{l}\text { Programa "EDFI" - Actividades basadas } \\
\text { en juegos }\end{array}$ & Infantil \\
\hline Larraz y Allueva (2012) & Habilidades creativas & $\begin{array}{l}\text { Actividades basadas en la generación } \\
\text { de ideas y resolución de problemas }\end{array}$ & Secundaria \\
\hline $\begin{array}{l}\text { Garaigordobil y } \\
\text { Berrueco (2011) }\end{array}$ & Creatividad gráfica y verbal & Sesiones de juego cooperativo-creativo & Infantil \\
\hline Barrena y Molina (2010) & $\begin{array}{l}\text { Creatividad y educación en } \\
\text { valores }\end{array}$ & Técnicas creativas y cuentos & Secundaria \\
\hline $\begin{array}{l}\text { López y Navarro- } \\
\text { Lozano (2010) }\end{array}$ & Habilidades creativas & $\begin{array}{l}\text { Actividades basadas en resolución de } \\
\text { tareas }\end{array}$ & Primaria \\
\hline
\end{tabular}




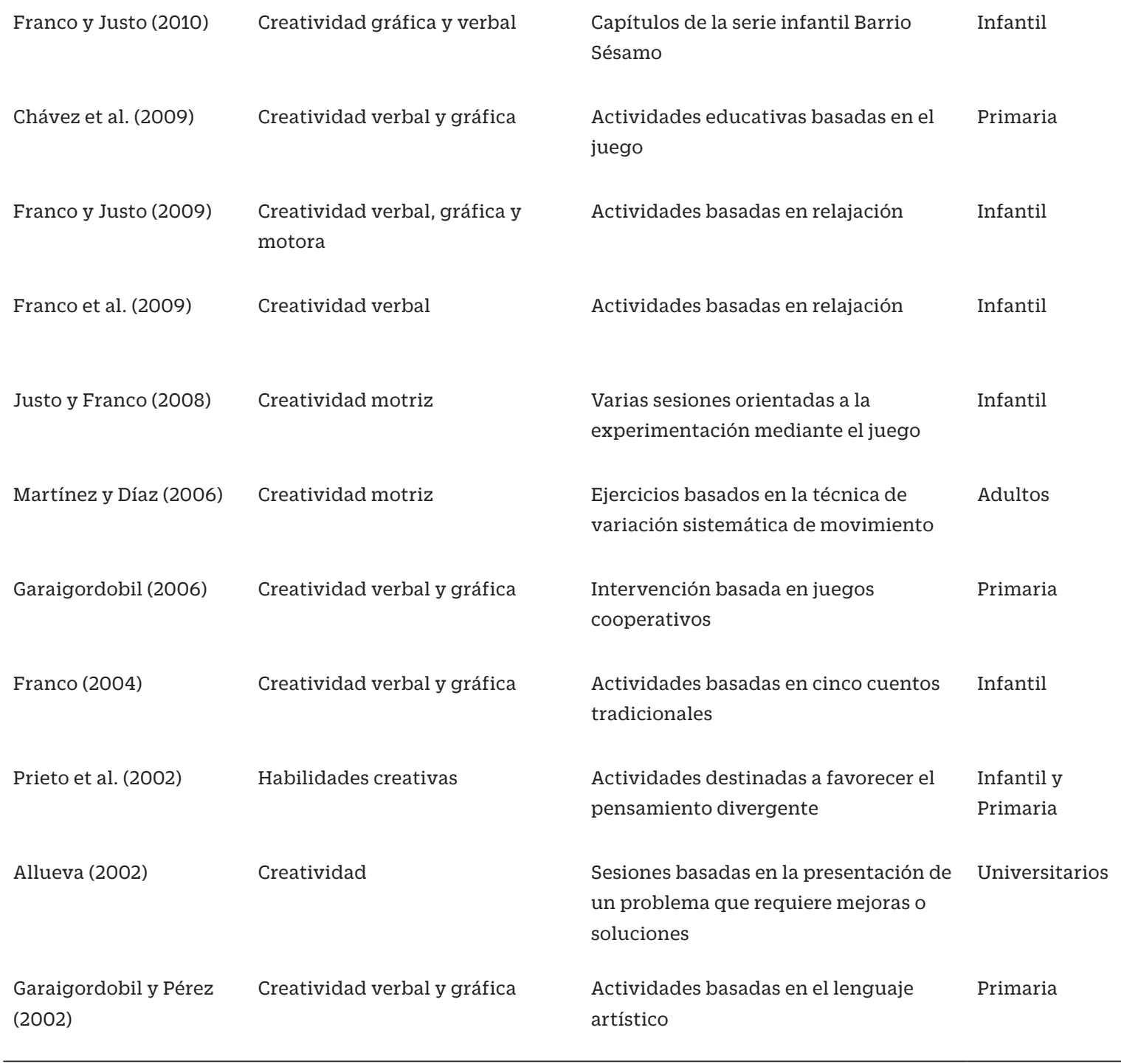

Para finalizar, es necesario recalcar la existencia de programas que tienen como finalidad contribuir a la formación y desarrollo de los jóvenes mediante la adquisición de ciertas capacidades como la creatividad (Olivos et al., 2013).

\section{Discusión}

La creatividad, aunque no tiene una definición exhaustiva, es entendida como el proceso de toma de decisiones que se realiza con una finalidad concreta mediante la imaginación y que tie- ne unos resultados observables en el mundo físico (Al-Ababneh, 2020; Cuetos et al., 2020).

Aunque la creatividad siempre ha estado presente en nuestras vidas, son muchos los investigadores que, en los últimos años, han sido atraídos por este constructo (Fernández et al., 2019; Morales-Valiente, 2017); ya que tal y como indica Bonilla (2015) y Castillo-Delgado et al. (2016) la creatividad está relacionada con el potencial humano y, con ello, con la capacidad de solucionar problemas (Fernández et al., 2019). Por ello, son muchos los estudios que se pueden encontrar en los últimos años sobre la creatividad y su relación con otras variables como el procesamiento de la 
información (Hurtado et al., 2018); la edad (Medina-Sánchez et al., 2017); el rendimiento académico (Chiecher et al., 2018); el aprendizaje (Bernabeu \& Goldstein, 2016; Rodríguez, 2018); el talento humano (Acosta et al., 2016); entre otros, debido a que la creatividad puede expresarse en cualquier ámbito y/o contexto (Elisondo, 2015).

Ciertos investigadores han enfocado su trabajo en medir la creatividad que presenta una serie de individuos mediante la utilización de diversas pruebas; esta tarea es bastante compleja debido a que este término presenta diferentes rasgos cognitivos y de personalidad. Por ello, este tema conlleva cierta controversia y varios autores indican que la creatividad es un constructo que no se puede medir, al contrario que otros, que afirman que la creatividad sí puede ser medida y se puede trabajar mediante una intervención (Arévalo et al., 2015; Sánchez et al., 2019). Antes de valorar la creatividad de una persona hay que tener en cuenta una serie de detalles como la subjetividad y la experiencia, cómo las investigaciones pueden verse afectadas de manera negativa por diferentes variables como el ambiente y cómo es posible modificar la creatividad dependiendo de ciertos factores (Díaz \& Justel, 2019; González et al., 2020; Ricci, 2020; Rodríguez \& Luzardo, 2020).

La realización de esta revisión sistemática ha corroborado la gran variedad existente de instrumentos que miden la creatividad y de recursos o programas que se utilizan con la finalidad de trabajarla en diferentes contextos. Aunque los métodos enfocados en evaluar la creatividad son diversos, la mayoría de ellos se centran en evaluar la creatividad mediante la toma de soluciones (Guilford, 1950; Romo et al., 2016; Torrance, 1966), las acciones creativas empleadas (Elisondo, 2020), así como, la percepción sobre su propia aptitud creativa (Auzmendi et al., 1996). Algunos autores señalan que cuanto mayor sea la puntuación obtenida en las dimensiones de la creatividad mayor será la capacidad creativa del sujeto (Soto et al., 2015), mientras que otros autores indicen en que todas las dimensiones de la creatividad se relacionan entre sí (Kim et al., 2006) y que solo hay fijarse en el resultado final (Martínez, 2016) o en la secuencia de tareas utilizadas has- ta llegar al elemento creativo (Barraca et al., 2011; Martínez-Zaragoza \& Corbalán, 2002).

Hay que tener en cuenta que, según al contexto a evaluar, las dimensiones pueden variar como es el ejemplo de Ayala-Pérez (2013), que muestra que para medir la creatividad en diseño gráfico las dimensiones por evaluar son: novedad, comunicación y estética. Así como, tal y como ocurre con la escala elaborada por Soroa et al. (2015), la cual se centra en las dimensiones preparación, novedad y efectividad con la finalidad de conocer la capacidad que tienen los sujetos evaluados de expresar y experimentar emociones. Para medir la creatividad dentro del contexto laboral, se encuentran dos escalas con las que se pretende conocer las percepciones que tienen los empleados en cuanto a la creatividad llevada a cabo en el trabajo (Boada-Grau et al., 2014b), así como, percibir el potencial creativo por parte de los trabajadores en el trabajo (Boada-Grau et al., 2014a).

Atendiendo a la diversidad de recursos que se pueden encontrar con el propósito de trabajar la creatividad, cabe destacar cómo la mayoría son llevados a cabo en un contexto educativo y con sujetos de corta edad, es decir, estudiantes de educación infantil o primaria. Es necesario la existencia de programas mediante los que se desarrollen ciertas habilidades creativas con la finalidad de potenciar la formación de los estudiantes (Olivos et al., 2013). Muchos investigadores han optado por utilizar tareas relacionadas con la implicación de la inteligencia y los procesos cognitivos como son la elaboración de nuevos productos o la resolución de problemas (Alfonso-Benlliure et al., 2013; Allueva, 2002; López \& Navarro-Lozano, 2010; Piraquive-Peña et al., 2015; Larraz \& Allueva, 2012).

Por otro lado, otros estudios han optado por emplear el juego y/o otras actividades que impliquen movimiento por parte de los participantes; aunque la mayoría de dichos estudios están enfocados a potenciar la creatividad motriz (Justo \& Franco, 2008; Martínez \& Díaz, 2006; Salinas-López et al., 2015), también se han obtenido beneficios en la creatividad gráfica y narrativa (Aranda, 2012; Castillo et al., 2018; Chávez et al., 2009; Garaigordobil, 2006; Garaigordobil \& Berrueco, 2011; Ocampo et al., 2019). Además, ciertas 
técnicas relacionadas con la creación artística-literaria también han sido relevantes a la hora de potenciar la creatividad, ya que, mediante estos recursos se estimulan variables como la imaginación, la cual tiene una gran relación con la creatividad. Algunas de estas técnicas pueden ser la creación de estructuras mediante materiales naturales (Garaigordobil \& Pérez, 2002; Jenaro-Río et al., 2019), actividades que impliquen relajación (Arazola-Ruano, 2017; Franco et al., 2009; Franco \& Justo, 2009), así como la lectura, escritura o incluso el visionado de cuentos (Barrena \& Molina, 2010; Franco, 2004; Franco \& Justo, 2010; Segundo et al., 2020; Núñez-Gómez et al., 2020).

En definitiva, para concluir, es necesario resaltar la variedad de instrumentos existentes para medir la creatividad y de recursos, técnicas y/o actividades focalizadas en trabajar la creatividad con la finalidad de, en mayor o menor medida, fomentar dicho constructo en los sujetos. Atendiendo a las implicaciones prácticas que puede tener este trabajo para futuras investigaciones, se puede indicar que esta revisión sistemática serviría para que otros investigadores conozcan cuál o cuáles son los instrumentos que mejor se adaptan al estudio por realizar, así como, cuáles son los recursos o técnicas más adecuados para la realización de una intervención. En cuanto a las limitaciones encontradas en la ejecución de este trabajo son debidas a que algunos estudios han tenido que ser descartados por no haber podido obtener el texto completo del mismo. Por otro lado, se considera de interés, para futuras líneas de investigación, atender de forma más específica a características que se consideran relevantes para el análisis de los instrumentos identificados en esta revisión, como su validez o confiabilidad. Aun así, esta revisión cuenta con una gran diversidad de instrumentos con los que se puede medir la creatividad y de recursos que han obtenido una puntuación significativa a la hora de potenciar la creatividad en sujetos de habla hispana.

Agradecimientos: El presente trabajo cuenta con el apoyo del Ministerio de Educación y Formación Profesional a través del programa de ayudas para la Formación de Profesorado Universitario (FPU) otorgado a Alba González Moreno con referencia FPU19/01570.

\section{Referencias}

Acosta, D., Batista, N., \& Suárez, L.A. (2016). La creatividad y el desarrollo del talento humano. Magazine de las ciencias: Revista de Investigación e Innovación, 1(2), 17-24. https://core.ac.uk/download/ pdf/235501583.pdf

Al-Ababneh, M. (2020). The concept of creativity: definitions and theories. International Journal of Tourism \& Hotel Business Management, 2(1), 245-249. https:// www.scitcentral.com/article.php?journal=46\&article=998\&article_title=THE\%20CONCEPT\%200F\%20 CREATIVITY:\%2ODEFINITIONS\%2OAND\%2OTHEORIES

Alexander, P.A. (2020). Methodological guidance paper: The art and science of quality systematic reviews. Review of Educational Research, 90(1), 6-23. https:// doi.org/10.3102\%2F0034654319854352

*Alfonso-Benlliure, V., Melendez, J.C., \& García-Ballesteros, M. (2013). Evaluation of a creativity intervention program for preschoolers. Thinkings Skills and Creativity, 10, 112-120. https://doi.org/10.1016/j. tsc.2013.07.005

*Allueva, P. (2002). Desarrollo de la creatividad: diseño y evaluación de un programa de intervención. Persona: Revista de la Facultad de Psicología, 5, 67-81. https://doi.org/10.26439/persona2002.n005.843

*Aranda, J. (2012). Programa "EDFI" y creatividad en pre escolares de 5 años de la zona rural de Pichanaki. Horizonte de la Ciencia, 2(3), 91-96. http://revistas. uncp.edu.pe/index.php/horizontedelaciencia/article/view/170

*Arazola-Ruano, C. (2017). Efectos de un programa de relajación creativa sobre los niveles de creatividad motriz en Educación Infantil. MLS-Educational Research, 1(1), 53-68. https://doi.org/10.29314/mlser.v1i1.18

Arévalo, M.P., Ezquerro, A., Arias-Castro, C.C., \& López-Fernández, V. (2015). Un análisis del constructo creatividad en función del instrumento de medida en adultos. E-Innova: Revista Electrónica de Educación. http://webs.ucm.es/BUCM/revcul/e-learning-innova/195/art2854.pdf

*Auzmendi, E., Villa, A., \& Abedi, J. (1996). Reliability and validity of a newly constructed multiple-choice creativity instrument. Creativity Research Journal, 9(1), 89-95. https://doi.org/10.1207/s15326934crj0901_8

Ayala-Pérez, J.L. (2013). Creación de un instrumento para medir la creatividad en diseño gráfico. Gráfica: Revista de Diseño Gráfico, 1(1), 23-39. https://raco.cat/ index.php/Grafica/article/view/283793

*Barraca, J., Ancillo, I., Artola, T., Mosteiro, P., Poveda, B., \& Sánchez, N. (2011). La prueba de imaginación creativa para adultos (PIC-A). Un nuevo instrumento para la evaluación del pensamiento divergente. Prolepsis, 9, 46-55.

*Barrena, J., \& Molina, M.A. (2010). Desarrollo de la creati- 
vidad desde un programa de animación a la lectura en la educación de valores. Trances, 2(5), 381411. https://revistatrances.wixsite.com/trances/ copia-de-2011

Bernabeu, N., \& Goldstein, A. (2016). Creatividad y aprendizaje: El juego como herramienta pedagógica ( $2^{\mathrm{a}}$ ed.). Narcea Ediciones.

*Boada-Grau, J., Sánchez-García, J.C., Prizmic-Kuzmica, A.J., \& Vigil-Colet, A. (2014a). Spanish adaptation of the Creative Potential and Practised Creativity scale (CPPC-17) in the workplace and inside the organization. Psicothema, 26(1), 55-62. https://doi. org/10.7334/psicothema2013.68

*Boada-Grau, J., Vigil-Colet, A., Sánchez-García, J.C., \& Prizmic-Kuzmica, A.J. (2014b). Adaptation of the Creative Environment Perceptions Scale into Spanish. Universitas Psychologica, 13(4), 1357-1366. https:// doi.org/10.11144/Javeriana.UPSY13-4.aece

Bonilla, A. (2015). Diseño de juegos y creatividad: un estudio en el aula universitaria. Opción, 31(4), 106-126. https://produccioncientificaluz.org/index.php/opcion/article/view/20541

Castillo-Delgado, M., Ezquerro-Cordón, A., Llamas-Salguero, F., \& López-Fernández, V. (2016). Estudio neuropsicológico basado en la creatividad, las inteligencias múltiples y la función ejecutiva en el ámbito educativo. Reidocrea, 5(2), 9-15. https://doi. org/10.30827/Digibug.39528

*Castillo, M.C., Caurcel, M.J., \& Chacón, H. (2018). Diseño, aplicación y efecto de un programa para la mejora de la creatividad en Educación Infantil. Revista Internacional de Apoyo a la Inclusión, Logopedia, Sociedad y Multiculturalidad, 4(3), 1-10. https://doi. org/10.17561/riai.v4.n3.1

*Chávez, B.I., Zacatelco, F., \& Acle, G. (2009). Programa de enriquecimiento de la creatividad para alumnas sobresalientes de zonas marginadas. Electronic Journal of Research in Educational Psychology, 7(18), 849-876. http://dx.doi.org/10.25115/ejrep.v7i18.1366

Chiecher, A.C., Elisondo, R., Paoloni, P.V., \& Donolo, D.S. (2018). Creativity, Gender and Academic Performance in Students Who Enter the Engineering Degree. Revista Iberoamericana de Educación Superior, 9(24), 138-151. http://www.scielo.org.mx/scielo.php?scrip$\mathrm{t}=\mathrm{sci}$ _arttext\&pid=S2007-28722018000100138\#aff4

Cuetos, M.J., Grijalbo, L., Argüeso, E., Escamilla, V., \& Ballesteros, R. (2020). Potencialidades de las TIC y su papel fomentando la creatividad: percepciones del profesorado. RIED: Revista Iberoamericana de Educación a Distancia, 23(2), 287-306. http://dx.doi. org/10.5944/ried.23.2.26247

Díaz, V., \& Justel, N. (2019). Creatividad. Una revisión descriptiva sobre nuestra capacidad de invención e innovación. CES Psicología, 12(3), 35-49. https://doi. org/10.21615/cesp.12.3.3

Elisondo, R. (2015). La creatividad como perspectiva educativa. Cinco ideas para pensar los contextos creati- vos de enseñanza y aprendizaje. Revista Electrónica Actualidades Investigativas en Educación, 15(3), 1-23. https://doi.org/10.15517/aie.v15i3.20904

*Elisondo, R. (2020). Creative Actions Scale: A Spanish Scale of Creativity in Different Domains. The Journal of Creative Behavior, 55(1), 215-227. https://doi. org/10.1002/jocb.447

Fernández, J.R., Llamas, F., \& Gutiérrez-Ortega, M. (2019). Creatividad: Revisión del concepto. Reidocrea, 8(37), 467-483. http://dx.doi.org/10.30827/Digibug. 58264

*Franco, C. (2004). Aplicación de un programa psicoeducativo para fomentar la creatividad en la etapa de Educación Infantil. Revista Electrónica de Investigación y Evaluación Educativa, 10(2), 245-266. https://doi.org/10.7203/relieve.10.2.4328

*Franco, C., \& Justo, E. (2010). “Sesame Street" and children's creativity: effects of a psychoeducational programme. Bordón- Revista de Pedagogía, 62(4), 81-94. https://recyt.fecyt.es/index.php/BORDON/article/ view/29320

*Franco, C., Justo, E., \& Fernández, J. (2009). Aplicación de un programa de relajación e imaginación para niños en Educación Infantil y su incidencia sobre sus niveles de creatividad verbal. Bordón - Revista de Pedagogía, 61(3), 47-57. https://recyt.fecyt.es/index. php/BORDON/article/view/28754

*Franco, C., \& Justo, E. (2009). Efectos de un programa de intervención basado en la imaginación, la relajación y el cuento infantil, sobre los niveles de creatividad verbal, gráfica y motora en un grupo de niños de último curso de educación infantil. Revista Iberoamericana de Educación, 49(3), 1-11. https:// rieoei.org/RIE/article/view/2095

*Garaigordobil, M. (2006). Intervention in Creativity With Children Aged 10 and 11 Years: Impact of a Play Program on Verbal and Graphic-Figural Creativity. Creativity Research Journal, 18(3), 329-345. https://doi. org/10.1207/s15326934crj1803_8

*Garaigordobil, M., \& Berrueco, L. (2011). Efectos de un programa de juego en el pensamiento creativo de los niños en edad preescolar. Revista Española de Psicología, 14(2), 608-618. https://doi.org/10.5209/ rev_SJOP.2011.v14.n2.9

*Garaigordobil, M., \& Pérez, J.I. (2002). Efectos de la participación en el programa de arte Ikertze sobre la creatividad verbal y gráfica. Anales de Psicología, 18(1), 95-110. https://revistas.um.es/analesps/article/view/28631

González, E., Pacheco, M., \& de Frutos, B. (2020). Dimensiones en la evaluación de la creatividad en campañas de comunicación integrada. Una aportación para la evaluación en el entorno docente. Doxa Comunicación, 30, 283-307. https://doi.org/10.31921/doxacom.n30a15

Guilford, J.P. (1950). Creativity. The American Psychologist, 5(9), 444-454. http://dx.doi.org/10.1037/h0063487 
Hurtado, P.A., García, M., Rivera-Porras, D.A., \& Forgiony-Santos, J.O. (2018). Las estrategias de aprendizaje y la creatividad: una relación que favorece el procesamiento de la información. Revista Espacios, 39(17), 12-30. https://www.revistaespacios.com/ a18v39n17/18391712.html

*Jenaro-Río, C., Castaño-Calle, R., \& García-Pérez, A. (2019). La experiencia de un taller para el fomento de la creatividad en niños de Primaria. Arte, individuo y sociedad, 31(4), 735-752. https://doi.org/10.5209/ aris.60841

*Justo, E., \& Franco, C. (2008). Influencia de un programa de intervención psicomotriz sobre la creatividad motriz en niños de educación infantil. Bordón - Revista de Pedagogía, 60(2), 107-121. https://recyt.fecyt. es/index.php/BORDON/article/view/29018

Kim, K.H., Cramond, B., \& Bandalos, D.L. (2006). The latent structure and measurement invariance of scores on the TTCT of Creative Thinking-Figural. Educational and Measurement, 66, 459-477. https://doi. org/10.1177/0013164405282456

*Larraz, N., \& Allueva, P. (2012). Efectos de un programa para desarrollar habilidades creativas. Revista Electrónica de Investigación en Psicología Educativa, 10(28), 1139-1158. http://dx.doi.org/10.25115/ejrep. v10i28.1553

*López, O., \& Navarro-Lozano, J. (2010). Influencia de una metodología creativa en el aula de primaria. European Journal of Education and Psychology, 3(1), 89102. https://doi.org/10.30552/ejep.v3i1.47

*Martínez, A., \& Díaz, M.P. (2006). The efficacy of a programme for improving the motor creativity of psychically disabled people. Apunts. Educación Física y Deportes, 84, 13-21. https://revista-apunts. com/eficacia-de-un-programa-de-intervencion-para-la-mejora-de-la-creatividad-motriz-en-un-grupo-de-discapacitados-psiquicos/

*Martínez-Zaragoza, F., \& Corbalán, F.J. (2002). Características psicométricas del CREA - Inteligencia Creativa. Un estudio con población española y argentina. Revista Iberoamericana de Diagnóstico y Evaluación Psicológica, 14(2), 103-115.

Martínez, Y. (2016). Dimensions and indicators for the development of creativity in the professional of Physical Culture. PODIUM: Revista de Ciencia y Tecnología en la Cultura Física, 11(3), 144-150. http://podium. upr.edu.cu/index.php/podium/article/view/669

Medina-Sánchez, N., Velázquez- Tejeda, M.E., Alhuay-Quispe, J., \& Aguirre-Chávez, F. (2017). La creatividad en los niños de prescolar, un reto de la educación contemporánea. REICE: Revista Iberoamericana sobre Calidad, Eficacia y Cambio en Educación, 15(2), 153181. https://doi.org/10.15366/reice2017.15.2.008

Morales-Valiente, C. (2017). La creatividad, una revisión científica. Arquitectura y Urbanismo, 38(2), 18155898. https://rau.cujae.edu.cu/index.php/revistaau/article/view/420
*Núñez-Gómez, P., Cutillas-Navarro, M.J., \& Álvarez-Flores, E.P. (2020). Cine como herramienta para el aprendizaje creativo en la escuela primaria. Es tudios sobre Educación, 38, 233-251. https://doi. org/10.15581/004.38.233-251

*Ocampo, A., Tovar-Cuevas, J.R., \& Arteaga-Díaz, G. (2019). The Game and Creative Cognition. A Proposal of Intervention. Psicología Educativa, 25(1), 59-65. https://doi.org/10.5093/psed2018a21

*Olivos, F., Álvarez, I., \& Díaz, F. (2013). Impacto de la educación para el emprendimiento en la creatividad: Una experiencia en Chile con Propensity Score Matching. Revista Electrónica Educare, 17(3), 259-276. https://doi.org/10.15359/ree.17-3.12

*Piraquive-Peña, C.J., López-Fernández, V., \& Llamas-Salguero, F. (2015). El uso del Tangram como estrategia de aprendizaje para el desarrollo de la creatividad y las inteligencias múltiples. Reidocrea, 4, 74-84. http://dx.doi.org/10.30827/Digibug.36548

*Prieto, M.D., López, O., Bermejo, M.R., Renzulli, J., \& Castejón, J.L. (2002). Evaluación de un programa de desarrollo de la creatividad. Psicothema, 14(2), 410-414. http://www.psicothema.com/psicothema. asp?id=741

Ricci, P. (2020). Una revisión general sobre la creatividad. Revista Científica Arbitrada de la Fundación MenteClara, 5, 1-12. https://doi.org/10.32351/rca.v5.201

Rodríguez, P., \& Luzardo, M. (2020). Cómo asegurar evaluaciones válidas y detectar falseamiento en pruebas a distancia síncronas. Revista Digital de Investigación en Docencia Universitaria, 14(2), e1240. https://doi.org/10.19083/ridu.2020.1240

Rodríguez, Y.V. (2018). Diseño de Serious Game para la enseñanza de la Responsabilidad Social en la Educación Superior. Revista Digital de Investigación en Docencia Universitaria, 12(1), 156-175. https://doi. org/10.19083/ridu.12.719

*Romo, M., Alfonso-Benlliure, V., \& Sánchez-Ruiz, M.J. (2016). The child creativity test (TCI): Assessing creativity through a problem finding task. Psicología Educativa, 22(2), 93-101. https://doi.org/10.1016\%2Fj. pse.2016.01.005

*Salinas-López, J.C., Espada, M., \& García-Coll, V. (2015). Una propuesta en Educación Física para el desarrollo de la creatividad en alumnos de tercero de primaria. ATHLOS. Revista Internacional de Ciencias Sociales de la Actividad Física, el Juego y el Deporte, 8, 47-68. http://museodeljuego.org/athlos-revista/ athlos-no8/una-propuesta-en-educacion-fisica-para-el-desarrollo-de-la-creatividad-motriz-en-alumnos-de-tercero-de-primaria/

Sánchez, J., Correa, P.S., \& Díaz, I.D. (2019). Revisión de las intervenciones que mejoran la utilidad percibida del aprendizaje de los estudiantes. Revista Digital de Investigación y Docencia Universitaria, 13(2), 45-56. https://doi.org/10.19083/ridu.2019.1077

*Segundo, R.I., López-Fernández, V., Daza-González, M.T., 
\& Phillips-Silver, J. (2020). Promoting children's creative thinking through reading and writing in a cooperative learning classroom. Thinkings Skills and Creativity, 36, 100663. https://doi.org/10.1016/j. tsc. 2020.100663

*Soroa, G., Gorostiaga, A., Aritzeta, A., \& Balluerka, N. (2015). A Shortened Spanish Version of the Emotional Creativity Inventory (the ECI-S). Creativity Research Journal, 27(2), 232-239. https://doi.org/10.10 80/10400419.2015.1030313

Soto, G., Ferrando, M., Sáinz, M., Prieto, L., \& Almeida, L. (2015). Creatividad y sus dimensiones: ¿de qué hablamos y qué evaluamos? Universitas in Psy- chologica, 14(3), 15-24. https://www.academia. edu/38729191/Creatividad_y_sus_dimensiones_De qu\%C3\%A9_hablamos_y_qu\%C3\%A9_evaluamos Creativity_and_its_Dimensions_What_we_Talk_ about_and_What_we_Evaluate

Torrance, E.P. (1966). Torrance Tests of Creative Thinking (Prueba de Torrance sobre Pensamiento Creativo). Personnel Press.

Urrútia, G., \& Bonfill, X. (2010). Declaración PRISMA: una propuesta para mejorar la publicación de revisiones sistemáticas y metaanálisis. Medicina clínica, 135(11), 507-511. https://doi.org/10.1016/j.medcli.2010.01.015

RIDU / Revista Digital de Investigación en Docencia Universitaria / e-ISSN: 2223-2516

(c) Los autores. Este artículo es publicado por la Revista Digital de Investigación en Docencia Universitaria del Área de Institutional Research and Effectiveness de la Dirección de Aseguramiento de la Calidad, Universidad Peruana de Ciencias Aplicadas. . "Este es un artículo de acceso abierto, distribuido bajo los términos de la LicenciaCreativeCommons Atribución 4.0 Internacional (CC BY 4.0) (https://creativecommons.org/licenses/by/4.0/deed.es), que permite el uso, distribución y reproducción en cualquier medio, siempre que la obra original sea debidamente citada." 